\title{
Les mesures financières hors normes - La profession comptable pourrait bien détenir la solution
}

\author{
Isabelle Lemay ${ }^{a}$, Daniel Tremblay ${ }^{b}$
}

RÉSUMÉ. Les mesures financières hors normes que l'on retrouve en marge des états financiers et qui ne sont donc ni régies par la normalisation comptable, ni couvertes par l'audit indépendant jouissent depuis plusieurs années d'une popularité grandissante. L'investisseur moyen ne pouvant faire la différence entre les données tirées des états financiers audités par des experts-comptables indépendants et toutes les autres informations rendues publiques par les directions d'entités et les médias, il nous apparât que la normalisation comptable devrait être plus proactive; notamment en améliorant la présentation des états financiers traditionnels de telle sorte à faire ressortir les différents éléments recherchés par les utilisateurs à travers ces mesures alternatives. Il nous semble évident qu'une présentation plus standardisée favorisera la comparabilité d'une entité à l'autre, mais aussi d'une période à l'autre. La profession comptable pourrait ainsi contribuer à limiter les comportements opportunistes de certains dirigeants et s'assurerait de remplir sa mission première, soit la protection du public.

ABSTRACT. Non GAAP financial measures that are reported outside of the traditional financial statements are not governed by official accounting standards and they are outside of the independent auditor purview. For every day investors, it has become very difficult to distinguish between financial information that comes from audited financial statements and all the other financial information made otherwise public by management or media. It is our view that standard organisations should be more proactive in this matter by simply rearranging the presentation of financial statements in a way that would emphasise the information sought by investors in those alternative measures. There is little doubt that more standardization of those alternative measures will enhance comparability between entities and also through time. In regard to non GAAP financial measures, we believe that the accounting profession could be an essential part of the effort to minimize opportunistic behavior by management and in this endeavour, it would contribute significantly to its primarily goal to protect the public.

\section{Introduction}

La normalisation comptable n'a jamais prétendu que les états financiers à vocation générale, soit les données financières préparées conformément aux principes comptables généralement reconnus (PCGR), étaient à eux seuls suffisants pour optimiser les décisions d'investissement ${ }^{1}$. Malgré cet aveu, la popularité grandissante des mesures financières présentées en marge des états financiers à vocation générale et qui ne sont donc ni régies par la normalisation comptable, ni couvertes par l'audit indépendant, s'avère une préoccupation constante pour tous les acteurs des marchés financiers, incluant les organismes de réglementation et les organismes de normalisation comptable.

Le phénomène n'est pas nouveau, ni d'ailleurs la préoccupation des organismes de réglementation. Déjà au début des années 1970, l'autorité des marchés financiers américains, la U.S Securities and Exchange Commission (SEC), avait soulevé des réserves quant à l'utilisation de telles mesures, mais il lui aura fallu près de 30 ans pour finalement intervenir un tant soit peu. La normalisation comptable, quant à elle, reste pour l'instant en marge du réel débat,

\footnotetext{
${ }^{a}$ Professeure, M. fisc., CPA auditeur, CA, Université du Québec à Chicoutimi

b Professeur, MPA, CPA auditeur, CA, Université du Québec à Chicoutimi
} 
mais cette attitude peut-elle se poursuivre encore longtemps si la profession veut demeurer pertinente? Certes, l'International Accounting Standards Board (IASB), organisme de normalisation international comptable, observe et commente à l'occasion sur le sujet, mais à ce jour, aucune proposition concrète n'a été présentée pour pallier les manques apparents des états financiers à vocation générale.

Les mesures financières hors normes (MFHN) désignent toutes les données quantitatives concernant la performance financière ou la situation financière d'une entité, mais qui ne sont pas dans les états financiers conformes aux PCGR. Ces mesures sont publiées par les entités (pro forma) dans leurs rapports annuels, dans leurs communiqués de presse ou sur leurs sites Web, mais on les retrouve aussi parfois dans les analyses d'experts financiers (street earnings). Quoiqu'elles puissent parfois concerner la richesse des entités ou encore plus restrictivement leurs liquidités disponibles, la plupart représentent une mesure quelconque de rentabilité qui s'avère en fait un bénéfice "ajusté ». Le bénéfice calculé conformément aux PCGR est ainsi « ajusté » pour tenir compte de divers éléments lesquels, selon la direction de l'entité dans le cas des mesures pro forma ou selon les analystes dans le cas des street earnings, sont jugés non pertinents aux fins d'évaluer la performance financière de l'entité et ses perspectives d'avenir. On retrouvera souvent dans ces mesures le BAAII (bénéfice avant amortissement, intérêt et impôts) de loin le plus couramment utilisé, mais aussi le «bénéfice opérationnel » ou le «bénéfice avant charges spéciales» ou encore simplement le «bénéfice ajusté » pour n'en nommer que quelquesuns. Les ajustements ainsi appliqués au bénéfice net calculé selon les PCGR sont très diversifiés, mais ils concernent le plus fréquemment l'un ou l'autre des éléments suivants :

1) Charges liées à la structure de financement (intérêts et autres frais financiers) et aux impôts.

2) Éléments hors trésorerie (amortissement, dépréciation, radiation d'inventaire).

3) Éléments non récurrents (résultats attribuables aux restructurations, aux regroupements d'entités ou à d'autres opérations similaires).

4) Éléments spéciaux (variations de valeur, incluant les gains et pertes sur disposition d'actifs, frais de recherche, frais de campagne publicitaire, indemnités issues de poursuites, charges liées aux rémunérations à base d'actions ou aux régimes d'avantages postérieurs à la retraite).

Ces ajustements auront généralement un impact important sur la mesure des résultats et force est de constater que l'effet est la plupart du temps positif 2 . À titre d'exemple, le rapport annuel de Bombardier en 2016 présentait les ajustements suivants pour démontrer notamment que l'entité avait atteint et même dépassé ses objectifs (en millions de dollars américains) ${ }^{3}$.
Résultat net selon les états financiers conformes aux PCGR Charges nettes de financement

Charge d'impôts

Amortissement

Éléments spéciaux

Avions C Series - Contrats déficitaires, dépréciation et autres Avions CRJ1000 - Charge de dépréciation

Learjet - Charge de dépréciation et autres charges

Charges de restructuration

Charges liées aux avantages de retraite

Modification des estimations et de la juste valeur

Litige fiscal

Radiation de frais différés

Résiliation d'ententes

Gain de change lié à la vente d'une participation minoritaire

RAIIA ajusté

\begin{tabular}{cc}
$\begin{array}{c}\text { Exercices clos les } 31 \\
\text { décembre } \\
\underline{\mathbf{2 0 1 6}}\end{array}$ & $\underline{\underline{\mathbf{2 0 1 5}}}$ \\
$(981) \$$ & $(5340) \$$ \\
174 & 571 \\
445 & 154 \\
& 501 \\
492 & 3235 \\
& 243 \\
$(59)$ & 1216 \\
215 & 9 \\
$(139)$ & 312 \\
& 50 \\
14 & 194 \\
& 133 \\
$(38)$ & \\
\hline $943 \$$ & $1278 \$$
\end{tabular}


Les ajustements effectués auront ainsi permis un redressement "positif» de près de 2 milliards de dollars (6,6 milliards en 2015), ce qui modifie considérablement le portrait financier de l'entité. De tels ajustements sont aujourd'hui monnaie courante et pour s'en convaincre, il suffit de constater l'état actuel du phénomène dans toute l'Amérique du Nord où le nombre d'entités qui n'utilisent pas les MFHN fait maintenant figure d'exception'.

Tous ces efforts déployés par les entités et les analystes pour calculer des mesures alternatives du bénéfice semblent fort révélateurs. Ils confirment dans un premier temps l'importance accordée par les marchés financiers aux résultats générés par une entité, et ce, non seulement dans leur globalité, mais également dans les divers éléments qui les composent (Burgstahler et Dichev, 1997; Skinner et Sloan, 2002; Bartov et al., 2002; Barth et al., 1999; Graham et al., 2004; Demirakos et al., 2004; Barker Imam, 2008). Les MFHN qui décortiquent les résultats fournissent ainsi une information jugée pertinente pour les investisseurs et à ce titre, la direction des entités, en présentant de tels ajustements peuvent certes se voir attribuer un objectif louable de communication à des fins de transparence. La prolifération des MFHN soulève également la question de la pertinence de l'approche PCGR qui a relayé au second plan le principe de rapprochement des charges aux produits pour favoriser une meilleure mesure de la richesse des entités (éléments présentés au bilan) $)^{5}$. Cette tendance de la normalisation comptable, dans laquelle s'inscrit de toute évidence une ouverture de plus en plus grande pour des mesures à la valeur marchande par opposition au coût historique, n'est certes pas un déni de l'importance du bénéfice net. En fait, la normalisation comptable serait plutôt d'avis que la meilleure mesure du bénéfice réalisé par une entité dans un exercice donné se trouve par l'écart dans sa richesse entre le début et la fin de cet exercice, dans la mesure évidemment où cette richesse est bien mesurée dans les bilans ${ }^{6}$. La popularité des MFHN et plus spécifiquement l'inventaire des bénéfices « ajustés » publiés à tous azimuts peuvent certainement semer un doute sur cette affirmation.

Qui plus est, la normalisation comptable favorise une approche de "résultat unique », soit une mesure de rendement qui tient compte de l'ensemble des revenus et des dépenses sans qualification spécifique de ces éléments de résultat à savoir s'ils constituent des éléments monétaires, s'ils sont liés à la structure de financement ou encore s'ils représentent des opérations courantes, opérationnelles ou autres. Encore une fois, l'utilisation répandue des MFHN qui permettent souvent de mettre en lumière la nature de plusieurs éléments spécifiques de résultats nous semble un indicateur fort qu'une remise en question de l'approche comptable actuelle pourrait être appropriée.

Évidemment, la volonté de communiquer des informations pertinentes deviendra parfois une simple excuse pour réorganiser les données financières afin de manipuler les marchés. Effectivement, la présentation des MFHN est indéniablement associée à des comportements opportunistes. Pour appuyer ce point, nous nous contenterons de relater la célèbre affaire Enron dont la chronologie des événements parle d'elle-même. Le 16 octobre 2001, dans une communication faite en marge des états financiers traditionnels, Enron déclarait un "bénéfice récurrent 》 par action dilué de $0,43 \$$ pour son $3 e$ trimestre (représentant une augmentation de $26 \%$ par rapport à la même donnée de l'année précédente) et faisait des prévisions à 1,80 \$ et à 2,15\$ pour les deux années à venir. En comparaison, le bénéfice par action calculé selon les PCGR était en fait une perte de 0,84 \$ montrant une situation détériorée par rapport à l'année précédente où la même donnée était de -0.34 \$. Lors d'une conférence de presse, la direction de l'entité expliquait que la différence entre la MFHN et la mesure PCGR était essentiellement attribuable à des charges non récurrentes qui, selon elle, " have clouded the performance and earnings potential of our core energy business ». Enron déclarait faillite six semaines plus tard.

Alors que la pertinence des MFHN semble bien fondée, il en est de même pour les comportements opportunistes qui y sont associés. Quoique la réglementation par les autorités responsables des marchés financiers ait eu certains impacts positifs en regard des MFHN, du moins à court terme, elle demeure clairement insuffisante. Dans ce contexte, on pourrait croire que la normalisation comptable pourrait apporter une contribution majeure à ce chapitre en proposant un modèle de calcul des résultats qui répond davantage aux besoins des marchés, c'est-à-dire en y intégrant des mesures à l'image des principales MFHN actuellement utilisées. L'intégration de telles mesures, dans le corps même des états financiers à vocation générale, permettrait de les assujettir non seulement à une normalisation plus stricte, leur assurant par le fait même une certaine standardisation, 
mais les soumettrait également à des procédures d'audit indépendant. Ultimement, nos travaux de recherche viseraient à déterminer si l'intégration des MFHN dans les états financiers à vocation générale pourra permettre d'améliorer la qualité des informations financières en limitant les comportements opportunistes, en favorisant une plus grande confiance des utilisateurs et en améliorant la transparence et la comparabilité des informations. Le présent article se veut alors une prémisse à de tels travaux afin d'en valider le cadre théorique et plus particulièrement les quatre assertions suivantes :

1) Le corps des états financiers est utilisé en priorité aux fins des décisions d'investissement (par opposition aux informations complémentaires présentées dans les notes aux états financiers).

2) Les MFHN présentent des informations pertinentes pour les investisseurs et pour les autres acteurs des marchés financiers (ces mesures présentent une valeur ajoutée par rapport aux états financiers traditionnels).

3) La qualité des MFHN est actuellement variable (manipulation, comparabilité, transparence, compréhensibilité).

4) La réglementation améliore la qualité des MFHN (permet de limiter les comportements opportunistes, améliore la confiance des utilisateurs et favorise la comparabilité) quoique dans son état actuel elle soit insuffisante.

En conclusion et en considérant la validité théorique de ces assertions, nous tenterons d'identifier, a priori, des éléments de solution qui pourraient être considérés notamment par les responsables de la normalisation comptable afin d'améliorer la présentation des informations financières présentées aux différents investisseurs.

\section{Utilité des états financiers traditonnels}

\subsection{Pertinence des états financiers}

Il y a quelques années, la profession comptable s'est dotée d'un objectif clair en regard des états financiers à vocation générale, à savoir de fournir une information utile pour la prise de décision en matière d'attribution des ressources par les apporteurs de capitaux. En d'autres termes et quoiqu'il puisse y avoir une multitude d'utilisateurs, les états financiers, dans leur ensemble, doivent aider principalement les investisseurs (créanciers ou actionnaires) actuels ou potentiels dans leurs décisions d'investissement ${ }^{7}$. À cet égard, il est important dans un premier temps d'évaluer si cet objectif est atteint, à savoir si les informations financières contenues dans les états financiers à vocation générale influencent réellement les décisions et dans l'affirmative, si les différentes façons de présenter ces informations, notamment si elles sont présentées dans le corps même des états financiers ou dans les notes complémentaires $^{8}$, peuvent modifier le jugement des décideurs. Bien que les MFHN ne soient pas présentées dans les états financiers traditionnels, il s'avère utile d'évaluer d'abord comment les parties prenantes utilisent les états financiers à des fins décisionnelles. Cette validation est nécessaire pour comprendre l'importance des informations comptables conformes aux PCGR, mais surtout pour en évaluer ensuite la pertinence relative par rapport aux MFHN.

Par ailleurs, afin de bien saisir la portée des constatations qui suivent, il est essentiel de bien comprendre les deux prémisses suivantes qui concernent la préparation des états financiers traditionnels :

- La responsabilité de préparer des états financiers conformes aux PCGR incombe à la direction de l'entité qui dispose d'une certaine latitude dans la présentation des informations. Ces choix laissés à la direction, qui pourront avoir des répercussions sur les montants constatés dans le corps des états financiers, mais aussi sur les montants et les informations présentés par voie de notes complémentaires aux états financiers, pourront affecter le jugement des utilisateurs. La direction détient alors un certain pouvoir d'influence sur l'information présentée dans les états financiers.

- Les notes complémentaires aux états financiers et qui en font partie intégrante contiennent une grande quantité d'informations, souvent complexes, mais non moins nécessaires à la compréhension des montants comptabilisés dans les états financiers ainsi que des éléments qui n'y sont pas comptabilisés. Ces notes donnent également des précisions sur certains postes des états financiers, ou encore elles rapprochent ou 
ventilent certaines informations. Elles servent en outre à expliquer les méthodes comptables utilisées, à fournir des informations sur les sources d'incertitude relatives aux estimations ainsi que des informations permettant aux utilisateurs d'évaluer les objectifs, procédures et processus de gestion du capital de l'entité9. Par conséquent, il n'est pas rare de constater que les notes complémentaires totalisent plusieurs dizaines de pages ${ }^{10}$ et constituent nécessairement la partie la plus complexe des états financiers.

Les états financiers sont couramment utilisés dans la prise de décision et la façon de présenter l'information financière a vraisemblablement un impact sur la perception des utilisateurs. En appui à cette affirmation, certains travaux de recherche suggèrent que les utilisateurs moins avertis tels que les investisseurs non professionnels seront davantage influencés par l'information pro forma; alors que les analystes professionnels (utilisateurs avertis) sauront mieux interpréter cette même information (Frederickson et Miller, 2004). Par contre, d'autres études mettent en évidence que la façon de dévoiler l'information financière a également un impact sur les jugements qu'apportent les analystes financiers spécialisés et que les notes complémentaires aux états financiers ne constituent pas un substitut adéquat à la constatation dans le corps des états financiers (Hirst et Hopkins, 1998, et Hirst et al., 2004). Ainsi, autant pour les utilisateurs qui travaillent régulièrement avec des états financiers, que ceux qui le font moins fréquemment, les différentes façons de présenter la même information sont susceptibles d'influencer leurs décisions.

Il nous semble important de constater dès lors que cette conclusion est en quelque sorte en contradiction avec la théorie de l'efficience des marchés qui suggère que le cours des actions reflète, jusqu'à un certain degré, l'information divulguée par voie de note aux états financiers. Les limites de la théorie des marchés efficients sont d'ailleurs clairement exprimées dans l'étude de Viger et al. (2005, p. 4) qui fait la constatation suivante :

[...] lorsqu'il s'agit du traitement de l'information financière, il existe un fossé entre la recherche fondée sur les modèles empiriques et la recherche expérimentale. Les modèles analytiques s'appuient sur l'hypothèse d'un marché efficient constitué de participants bien informés et rationnels et ils postulent que les choix comptables entre des formules alternatives de divulgation ne devraient pas influencer les jugements et les décisions des utilisateurs des états financiers à la condition que le contenu informatif soit identique [...] alors que les recherches expérimentales en arrivent à la conclusion que les utilisateurs des états financiers ne sont pas nécessairement efficients dans leur traitement de l'information.

Il est également intéressant de constater que les professionnels de crédit bancaire, qui sont généralement considérés comme des utilisateurs avertis, sont tout autant influencés par la façon dont l'information financière est présentée dans les états financiers. Par exemple, Viger et al. (2005) ont évalué la capacité des utilisateurs des états financiers à interpréter adéquatement l'information relative aux options d'achat d'actions (OAA), sujet pour lequel les dirigeants pouvaient à l'époque choisir le traitement comptable à savoir une charge à l'état des résultats ou une explication par voie de notes complémentaires. Les auteurs concluent que la façon dont les OAA sont divulguées influence significativement les jugements et les décisions des directeurs de comptes. Plus particulièrement, les banquiers évaluent la cote de risque de crédit à un niveau plus élevé et ont une tendance plus pessimiste lorsque la charge est comptabilisée dans l'état des résultats plutôt que d'être divulguée par voie de notes aux états financiers. Dans les mêmes circonstances, ils sont moins enclins à accorder un prêt et exigent une prime de risque plus élevée, car ils jugent moins favorables la situation financière globale de l'entreprise, sa capacité à maintenir sa croissance et sa capacité à rembourser ses dettes. Les auteurs dégagent ainsi un constat général à savoir que la divulgation par voie de notes ne peut effectivement être un substitut adéquat à la comptabilisation dans le corps même des états financiers. D'autres études rejoignent ces conclusions et montrent que l'information sera davantage prise en considération si elle est incluse dans le bilan que si elle est présentée par voie de notes aux états financiers (Harper et al., 1987) et que les banquiers plus spécifiquement, dans leurs calculs de ratios, ont tendance à ignorer 
l'information contenue dans les notes aux états financiers (Brooks et al., 1996).

Ces études, prises dans leur ensemble, confirment que les différentes façons de présenter l'information dans les états financiers influencent les jugements et évaluations des utilisateurs et plus particulièrement que les informations contenues dans le corps même des états financiers seront davantage considérées que celles présentées dans les notes complémentaires. Dans cette perspective, il nous apparaît évident que la normalisation comptable devrait favoriser la présentation des principales informations quantitatives dans le corps même des états financiers par opposition aux notes complémentaires. Une telle approche améliorera effectivement l'utilité des états financiers en respectant le fait que les utilisateurs, à l'exception d'une poignée d'experts, puissent avoir des difficultés à interpréter adéquatement l'information diffusée dans les notes complémentaires. Bien que chaque information présentée dans les notes puisse, en théorie, aider l'utilisateur à comprendre les états financiers, la valeur de cette information s'avère en réalité nettement diminuée par la capacité d'absorption et de compréhension de l'utilisateur moyen. L'ampleur et la complexité des notes complémentaires constituent vraisemblablement les principales raisons pour lesquelles la plupart des utilisateurs y accordent moins d'importance.

\subsection{Limites des états financiers}

De toute évidence et certainement à juste titre, les apporteurs de capitaux, qu'il s'agisse d'actionnaires ou de créanciers, doivent aller au-delà des seuls états financiers pour prendre des décisions éclairées. Une faiblesse importante concernant les informations présentées dans les états financiers est d'ailleurs qu'elles se limitent à des éléments essentiellement quantitatifs. En effet, les états financiers ne tiennent pas compte d'éléments non financiers considérés comme essentiels à la bonne conduite des affaires (par exemple, la satisfaction de la clientèle, le taux de roulement des employés, le style de gouvernance).

Pour reprendre l'exemple des banquiers, ces derniers disposent souvent de plusieurs informations (liste des comptes clients, liste de stocks, carnet de commandes, entrevues avec les dirigeants, etc.) pour faire un diagnostic complet de la situation financière de l'entité. Les établissements de crédit ont des normes à respecter et pratiquement aucun banquier, quel que soit son intérêt pour une entité, ne pourra la soutenir si elle ne remplit pas les critères fixés par l'institution financière. Plus précisément, les décisions de crédit se prennent sur la base d'informations financières quantifiables, telle l'analyse de ratios généralement calculés à partir des états financiers, mais aussi à partir d'informations de nature plutôt qualitative comme les caractéristiques de la gouvernance ou la réputation de l'entreprise (Scott, 2006). Le banquier joue un rôle clé dans l'interprétation des informations qualitatives afin de fournir un profil plus complet pour l'analyse de financement. Sans ces informations, plusieurs petites entreprises risquent de voir leur demande de financement rejetée du fait que les informations financières quantifiables sont limitées ou incomplètes (Berger et Udell, 1995). Les banquiers donnent une valeur ajoutée à l'information de nature qualitative puisqu'elle leur permet une certaine latitude d'interprétation contrairement à l'information quantitative dont la nature même impose une certaine rigidité (Scott, 2006). À l'instar des banquiers, les investisseurs sur le marché des capitaux pourront également avoir accès à certaines analyses qualitatives notamment à partir des informations contenues dans les rapports annuels des entités. Évidemment, comme ces investisseurs ne jouissent pas d'une proximité similaire à celle des créanciers et quoique certains analystes puissent avoir accès à quelques informations privilégiées, l'information reste nettement moins personnalisée.

Malgré ce qui précède, les données quantitatives demeurent encore fort recherchées par les investisseurs aux fins de leur processus décisionnel (Kwok, 2002; Kemp et al., 1990). Ainsi, quoiqu'on puisse leur greffer certains aspects plus qualitatifs pour mieux évaluer la situation financière réelle de l'entité, les données chiffrées demeurent convoitées et dans cette perspective, les états financiers traditionnels restent une source d'information de premier plan pour les investisseurs. On reproche toutefois également aux informations issues des états financiers traditionnels le fait qu'elles se limitent à relater des événements historiques et qu'elles ont ainsi peu de valeur prédictive. De plus, les états financiers conformes aux PCGR et qui sont par définition soumis à une normalisation stricte sont considérés comme souvent trop rigides pour bien refléter la réalité propre à chaque entité. Ainsi, les MFHN 
semblent s'inscrire dans cette perspective de fournir d'autres informations quantitatives offrant davantage de souplesse du fait qu'elles ne sont pas standardisées en plus d'être axées sur les perspectives d'avenir en faisant abstraction des événements passés qui ne seraient pas susceptibles de se répéter dans le futur.

\section{MFHN - Pertinence et qualité}

Plusieurs intervenants sur les marchés financiers estiment que les MFHN peuvent être un meilleur indicateur des résultats futurs et fournir de l'information plus pertinente que les mesures conformes aux PCGR. D'ailleurs, de nombreux investisseurs utilisent leurs propres paramètres d'analyse et ajustent en conséquence les résultats financiers aux fins d'évaluer les perspectives d'avenir d'une entité. Ils sont d'avis que des ajustements sont nécessaires pour réduire le plus possible les effets d'opérations qui ne sont pas reliées aux activités courantes de l'entité ou qui ne reflètent pas fidèlement la réalité commerciale sous-jacente et donc qu'ils sont moins pertinents pour les investisseurs ${ }^{11}$. Qui plus est, l'Autorité canadienne en valeurs mobilières (ACVM) reconnait même explicitement l'utilité des ajustements apportés aux résultats mesurés conformément aux PCGR :

[...] les mesures financières non conformes aux PCGR peuvent donner aux investisseurs de l'information supplémentaire leur permettant de mieux comprendre les principales composantes de la performance financière de l'émetteur ${ }^{12}$.

L'utilisation massive de ces informations par les analystes et les décideurs est une démonstration assez forte que les utilisateurs les jugent pertinentes dans leur processus décisionnel. Les organismes de réglementation mondiale ont d'ailleurs dû s'adapter au fil des ans afin d'admettre l'utilité de ces mesures. Ainsi, la SEC qui s'était historiquement montrée hostile à l'utilisation de MFHN accepte maintenant l'idée que ces dernières soient utilisées et reconnait qu'elles puissent fournir des informations pertinentes du moins depuis que des changements ont été apportés aux exigences de présentation (Capeci, 2010). L'Autorité européenne des valeurs mobilières et des marchés (AEMF) a également reconnu l'importance des ajustements apportés aux résultats standardisés ${ }^{13}$. Même le président de l'IASB admet que les utilisateurs puissent avoir besoin de MFHN pour raffiner leur évaluation de la situation financière de l'entreprise. Dans une allocution prononcée à Tokyo en février 2014, il reconnaît que le résultat déterminé selon les PCGR constitue une mesure fiable et robuste, mais qui manque parfois de nuance pour représenter adéquatement les performances de l'entreprise. Aucun chiffre à lui seul ne peut saisir toutes les variables explicatives de la situation financière et pour cette raison, il faut accepter que les utilisateurs puissent avoir besoin d'informations supplémentaires pour ajouter de la précision à leurs évaluations ${ }^{14}$.

Nous avons déjà établi que les données quantitatives étaient fortement convoitées dans les décisions d'investissement et que les différentes façons de présenter la même information financière notamment dans les états financiers modifient les signaux envoyés aux utilisateurs et influencent alors leurs perceptions et leurs jugements. Les MFHN constituent certes de telles données quantitatives, mais comme elles ne sont pas présentées dans le périmètre des états financiers et qu'en conséquence, elles ne sont pas soumises à un audit indépendant, la question demeure entière à savoir si ces mesures «alternatives » influencent la perspective des investisseurs et surtout si elles favorisent de «meilleures » décisions d'investissement. Plus particulièrement, il s'agit dans un premier temps d'évaluer si les investisseurs utilisent ces informations dans leur processus décisionnel, mais également de déterminer si l'information fournie s'avère effectivement plus pertinente ou de meilleure qualité à des fins décisionnelles ou encore si elle présente un niveau d'opportunisme évident.

\subsection{Utilité des MFHN dans le processus décisionnel}

Une information financière que l'on qualifie «d'utile » profitera à la fois aux investisseurs et aux émetteurs et elle est d'autant plus efficace qu'elle répond aux besoins de ces utilisateurs. Par contre, lorsqu'une entité choisit de fournir des informations différentes de celles que fournissent ses concurrents ou qui ne respectent pas les «standards» comptables, certains intervenants mettent alors en garde les investisseurs quant à l'utilité limitée de celles-ci. En effet, la présentation des données financières en «dehors des états financiers », l'absence d'uniformité de celles-ci et surtout la latitude absolue laissée aux directions des entités quant à la 
nature et à la quantité des informations ainsi présentées pourraient laisser croire que les investisseurs ne les prendront pas en considération dans leurs analyses et qu'en conséquence, elles ne sont que de peu d'utilité.

La littérature indique néanmoins le contraire, notamment parce que la valeur prédictive s'en trouverait améliorée par les ajustements effectués aux résultats publiés en conformité avec les PCGR. Par exemple, Graham et al. (2006) constatent que les gestionnaires considèrent les bénéfices pro forma comme l'un des indicateurs de performance les plus importants divulgués aux investisseurs. L'exclusion de certains éléments dans le calcul des résultats financiers est parfois nécessaire pour les utilisateurs afin qu'ils puissent déterminer plus facilement la nature et l'ampleur des variables qui contribuent à la performance financière des entreprises. Plusieurs études appuient l'idée que, dans leurs calculs de MFHN, les dirigeants et les analystes excluent couramment les éléments non récurrents ou non opérationnels, éléments qui sont généralement considérés comme un masque à la réelle performance financière d'une entité. Plus particulièrement, Brown et Sivakumar (2003) concluent que le résultat net déterminé selon les PCGR inclut plusieurs éléments qui ne sont pas reliés aux opérations courantes ce qui réduit la pertinence des informations par rapport aux résultats ajustés. En d'autres termes, les utilisateurs veulent évaluer la performance des entreprises en fonction d'événements qui sont susceptibles de se reproduire dans le futur et non seulement en fonction des événements historiques tels que rapportés dans les états financiers conformes aux PCGR. Par ailleurs, Bhattacharya et al. (2003) ont trouvé qu'en comparaison avec les informations issues des états financiers conformes aux PCGR, les résultats proforma procurent une information permettant de présenter des bénéfices qui se rapprochent davantage de ceux susceptibles de se reproduire dans le futur.

Mais l'inventaire des MFHN met aussi en évidence que parmi les ajustements faits par les entités, on retrouve plusieurs éléments récurrents standards tels que la charge d'amortissement, les frais d'intérêts, les impôts, les frais de recherche et développement et la rémunération à base d'actions. En agissant ainsi, les préparateurs auraient comme motif d'isoler les effets de certaines décisions prises par la direction de l'entité pour ne conserver que les résultats liés aux activités courantes propres au secteur d'activités, favorisant ainsi une meilleure comparaison avec les concurrents.

En matière d'utilisation des MFHN, force est de constater que les dirigeants ont tendance à mettre l'accent sur leurs MFHN lorsqu'ils annoncent les résultats périodiques d'une entité et les analystes et investisseurs répondent dans le même sens en se concentrant également davantage sur ces mesures dans leurs analyses (Bradshaw et Sloan, 2002). Il nous apparaît donc évident que les MFHN sont réellement utilisées dans le processus décisionnel, mais considérant l'importance des écarts entre ces mesures et les résultats présentés dans les états financiers traditionnels, il est impératif de déterminer si ces mesures alternatives sont de qualité suffisante pour réellement aider les investisseurs à prendre des décisions plus éclairées.

\subsection{Lacunes des MFHN}

Depuis plusieurs années, la normalisation comptable a identifié les qualités que devait avoir l'information financière afin d'être utile aux investisseurs ${ }^{15}$. Quoique ces principes fondamentaux ne soient officiellement applicables que pour les états financiers à vocation générale, la plupart des vertus auxquelles ils font référence peuvent certainement trouver leur place dans le contexte des MFHN. Comme mentionné précédemment, les MFHN trouveront vraisemblablement leur principal appui dans la valeur prédictive qu'elles représentent, élément fondamental à la pertinence des informations financières. Dans l'état actuel des choses, ces mesures pourront toutefois plus difficilement atteindre un niveau acceptable en matière de compréhensibilité et de comparabilité.

La présentation de MFHN pourrait certes contribuer à surcharger d'informations les investisseurs, ne serait-ce que du seul fait que plusieurs entités en présentent une quantité impressionnante et que de surcroît elles s'ajoutent aux données présentées dans les états financiers qui elles-mêmes, en tenant compte des notes complémentaires, pourront donner plus d'une centaine de pages d'information ${ }^{16}$. Une telle quantité d'information contribue souvent à noyer l'essentiel et pourra ainsi nuire à la prise de décisions optimales. Ces mesures peuvent également être difficiles à interpréter en raison de leur caractère non standard puisqu'il devient alors très 
laborieux de saisir le mode de calcul utilisé par une entité donnée.

Également, il est évident que dans le contexte actuel, ces mesures ne sont pratiquement jamais comparables puisque les entités peuvent les dessiner à leur gré et que leur mode de calcul peut être modifié d'une période à l'autre. La comparabilité est particulièrement importante dans le cadre des décisions d'investissements qui impliquent généralement un choix entre plusieurs entités. Comme les MFHN peuvent varier d'une entité à l'autre, il s'avère souvent très difficile, voire impossible de les comparer avec des mesures du même type, quoique souvent désignées par le même nom, mais présentées par d'autres entreprises. Par ailleurs, en favorisant le maintien de mesures stables dans le temps, des tendances à plus long terme peuvent être dégagées, ce qui pourra contribuer grandement à la valeur prédictive tant convoitée de l'information. Encore une fois, les MFHN ne sont pas du tout au rendez-vous à ce chapitre.

La présentation de rapprochements quantitatifs entre les informations diffusées selon les PCGR et les MFHN constitue probablement l'une des façons d'atténuer ces effets néfastes et la littérature vient appuyer cette affirmation. En effet, les erreurs de prédiction des résultats sont moins nombreuses lorsque les entreprises effectuent un meilleur rapprochement entre les résultats conformes aux PCGR et ceux établis selon des mesures hors normes (Zhang et Zheng, 2011). De plus, les résultats ajustés par les dirigeants influencent les investisseurs, mais cette influence est atténuée par la présence d'un rapprochement des résultats (Elliott, 2006). Finalement, Aubert et Grudnitski (2014) concluent que les écarts dans l'évaluation des résultats prévisionnels prévalent surtout lorsque les rapprochements entre les MFHN et celles conformes aux PCGR sont de piètre qualité.

Quoique les analyses démontrent clairement que les investisseurs jugent utiles les MFHN, il nous semble raisonnable de considérer cette constatation à la lumière des conciliations actuellement exigées et des détails supplémentaires que celles-ci fournissent nécessairement. Les ajustements effectués par les analystes et la direction dans leurs calculs des MFHN et qui doivent faire l'objet d'une conciliation explicite augmentent certainement la précision des informations financières diffusées et il est alors possible que les constatations quant à la pertinence des MFHN soient le fruit d'une meilleure qualité générale des informations communiquées par ces entités notamment par les rapprochements quantitatifs plutôt que par la présentation de ces mesures en tant que telle. En effet, les conclusions quant à la pertinence des MFHN sont tirées d'études qui en évaluent la qualité, mais uniquement lorsque les entités émettrices procèdent à des rapprochements quantitatifs et clairement, ces derniers aident à mieux interpréter les résultats financiers. Ainsi, il nous semble légitime à ce stade d'envisager la possibilité que la source réelle du problème soit le manque de précision des informations présentées dans les états financiers à vocation générale. Il s'agit d'une question que les organismes de normalisation comptable ont probablement soulevée au cours des dernières années, mais force est de constater qu'aucun changement n'a été recommandé à ce jour.

\subsection{Opportunisme des MFHN}

La conciliation entre les résultats conformes aux PCGR et ceux ajustés ne pourra évidemment permettre d'éliminer totalement le risque que ces mesures alternatives de rendement soient manipulées ou biaisées afin notamment de gonfler artificiellement les bénéfices ou diminuer les pertes. Devant toute la latitude accordée aux préparateurs de ces informations, il est pratiquement inévitable que certaines entités les définissent de manière opportuniste. À cet effet, la probabilité que les prévisions des analystes soient dépassées est plus grande, puisque les dirigeants jouissent d'une liberté totale quant au choix des ajustements qui leur permettront d'atteindre leurs cibles financières. (Doyle et al. 2013).

Plusieurs intervenants sont convaincus de l'utilité des MFHN, tout en reconnaissant que leur divulgation reflète les motivations stratégiques des gestionnaires afin notamment d'influencer l'opinion des investisseurs et des analystes quant à la performance financière de l'entité. Certaines entreprises octroient même des régimes de rémunération de hauts dirigeants basés sur les bénéfices ajustés ce qui pourra certainement inciter ces mêmes dirigeants à adopter des comportements opportunistes le moment venu de calculer des mesures alternatives. Sachant que les investisseurs moins sophistiqués dépendent fortement de l'information pro 
forma (Frederickson et Miller, 2004; Bhattacharya et al., 2007), ces utilisateurs sont sans doute les plus à risque d'être induits en erreur lorsque les ajustements sont considérés comme opportunistes.

L'opportunisme de gestion dans les rapports financiers et l'effet subséquent sur les perceptions des investisseurs sont amplement documentés dans les différents travaux de recherche. Par exemple, l'exclusion de charges récurrentes des bénéfices ajustés est motivée par les incitations des dirigeants à respecter et même à surpasser les cibles financières établies (Black et Christensen, 2009, Lougee et Marquardt, 2004). De plus, les entités qui n'atteignent pas les prévisions des analystes sur la base des résultats mesurés conformément aux PCGR ont tendance à utiliser davantage les MFHN (Bhattacharya et al., 2003 et Bhattacharya et al., 2004) ce qui suggère également un opportunisme de gestion; quoique certains dirigeants pourraient plaider la volonté de fournir une information plus représentative de la performance financière de l'entité. D'ailleurs, la littérature reconnaît la difficulté à distinguer l'opportunisme du désir de présenter une " meilleure » information. Certains auteurs dont Black et Christensen (2009) désignent l'opportunisme par des ajustements du bénéfice considérés comme "agressifs ${ }^{17}$ et donc potentiellement trompeurs. Par ailleurs, Marques (2006) indique que la réaction boursière aux ajustements sur lesquels les gestionnaires et les analystes sont en désaccord est nettement inférieure à la réaction aux ajustements sur lesquelles les gestionnaires et les analystes sont en accord. Ce résultat suggère que les investisseurs considèrent les exclusions supplémentaires des dirigeants (au-delà de celles faites par les analystes) comme une élimination inappropriée de dépenses récurrentes. Finalement, Brown et al. (2012a) constatent que les dirigeants accélèrent le moment de l'annonce des résultats financiers dans les trimestres pour lesquels les résultats sont ajustés par rapport aux trimestres où aucun ajustement n'est apporté. En outre, ils relèvent que l'accélération de l'annonce des résultats augmente avec l'ampleur de l'exclusion d'éléments récurrents et lorsque les rapprochements quantitatifs entre les résultats ajustés et ceux conformes aux PCGR sont de moindre qualité. On remarque donc un certain opportunisme de gestion, et ce, même dans la planification du moment où les MFHN sont diffusées.
Ces résultats permettent de reconnaître le caractère parfois opportuniste des MFHN, mais leur utilité ne peut être contestée. Parfois, elles peuvent conduire à de «mauvaises » décisions, mais plusieurs autres facteurs imprévisibles peuvent également en être responsables. Par exemple, des changements dans les conditions économiques du marché, une nouvelle réglementation dans le secteur d'activités, un changement dans la direction de l'entreprise ou toute autre situation imprévisible peuvent contribuer à ce que les prévisions ne se matérialisent pas. À cet égard, l'étude de Malone et al., 2016 montre que les erreurs dans les prévisions sont moindres dans l'exercice financier qui suit la divulgation des résultats ajustés, ce qui semble indiquer que les ajustements sont utiles plutôt qu'opportunistes.

\section{Réglementation \\ 3.1 Description de la réglementation adoptée}

L'utilisation des MFHN est dans la mire des organismes de réglementation depuis fort longtemps. En 1973, la SEC mettait déjà en garde les investisseurs devant le caractère potentiellement trompeur de telles mesures ${ }^{18}$. La prolifération de ces mesures durant les années 1990, jumelée à certains abus notoires et aux scandales financiers tels WorldCom et Enron, pour n'en nommer que quelques-uns, a forcé la SEC à intervenir officiellement. Le 16 janvier 2002, dans une première intervention notable et qui s'avérera fort révélatrice des mesures à venir, la SEC a donné un ordre de cessation et d'abstention contre la compagnie Trump Hotels \& Casinos Resorts Inc. en réponse aux résultats trimestriels annoncés par la compagnie. Les allégations de la SEC indiquaient que les résultats affichés étaient trompeurs du fait qu'ils faisaient abstraction d'une charge non récurrente alors que les revenus constatés selon les PCGR incluaient, sans le présenter distinctement, un gain non récurrent. Quoique la compagnie n'ait jamais admis ses torts dans cette affaire, les publications visées ont été retirées.

Faisant suite à la Loi Sarbanes-Oxley ${ }^{19}$ de 2002, la SEC a adopté, le 15 janvier 2003, le Règlement G ${ }^{20}$ et l'ACVM n'a pas tardé en emboîter le pas avec son Avis 52-306 émis en novembre 2003. Ces réglementations qui sont essentiellement similaires n'empêchent pas la publication des MFHN et elles ne prescrivent aucune mesure spécifique, se limitant à en 
encadrer la présentation. Les principales exigences de cette réglementation, qui a subi ponctuellement quelques améliorations, sont les suivantes :

- Indiquer que les mesures n’ont pas de sens normalisé par les PCGR et que la comparaison avec des mesures similaires présentées par d'autres entités pourrait être impossible.

- Nommer la mesure d'une façon qui n'est pas trompeuse et qui la distingue des mesures conformes aux PCGR.

- Expliquer l'utilité des mesures pour les investisseurs et, le cas échéant, les raisons pour lesquelles la direction de l'émetteur en fait usage.

- Présenter les mesures financières de l'émetteur conforme aux PCGR qui sont les plus directement comparables en les mettant autant ou davantage en évidence et fournir un rapprochement quantitatif clair entre celles-ci et les mesures financières hors norme.

- Veiller à ce que les mesures ne décrivent pas les ajustements comme non récurrents, rares ou inhabituels lorsqu'un élément semblable est raisonnablement susceptible de se produire dans les deux exercices suivants ou s'est produit au cours des deux exercices précédents.

- Présenter la mesure financière non conforme aux PCGR de façon constante d'un exercice à l'autre.

Ainsi, l'approche réglementaire choisie est essentiellement axée sur des éléments de présentation, d'explication et de conciliation. Évidemment, cette réglementation, qui entraîne une augmentation notable de l'information fournie en regard des MFHN21, pourra s'avérer un frein à la divulgation ou à la compréhension d'informations considérées pertinentes par la plupart des intervenants. Cette préoccupation a d'ailleurs été clairement exprimée dès la mise en place de la réglementation :

While the objectives of the Sarbanes-Oxley Act provision and the resulting SEC rules are admirable, their results may in many instances be more of a burden than a benefit to the investing public. (...) The new SEC rules do not probibit the use of most types of non-Gaap financial measures, and specifically do not prohibit disclosures of Ebitda. Therefore, they don't directly deprive investors of access to that information. But they will make investors wade through discussions of what investors may view as irrelevant comparable Gaap financial measures and management's views about the usefulness of particular non-Gaap financial information as the price of being given the information. (...) Whether the benefits of the new SEC rules will outweigh the extent to which they impede coherent disclosure remains to be seen (Bernstein, 2003, 1-3).

Quoi qu'il en soit, le message des autorités réglementaires ne devenait alors ni subtil ni ambigu. Ne cherchant d'aucune façon à limiter l'utilisation des MFHN et comme constaté précédemment, elles en reconnaissaient dès lors tacitement leur pertinence. Par ailleurs, l'absence d'une quelconque forme de standardisation des mesures permises valide l'importance de laisser aux entités la liberté de moduler leurs indicateurs de performance en fonction de leurs caractéristiques spécifiques. En effet, plusieurs sont d'avis que la direction de l'entité est la mieux placée pour identifier les ajustements appropriés dans leur contexte spécifique (Holtzman et al., 2003; Venter et al., 2014). À la lumière de la réglementation adoptée, l'objectif serait donc de limiter les comportements opportunistes, mais sans toutefois empêcher la présentation d'informations pertinentes.

\subsection{Impact de la réglementation}

Plusieurs études ont été réalisées dans les années qui ont suivi la mise en place de la réglementation américaine afin d'en mesurer les impacts. Un consensus clair semble alors se dégager voulant que la réglementation ait entraîné une diminution générale dans l'utilisation des MFHN ce qui implique non seulement une diminution du nombre d'entités qui publient des MFHN, mais également une diminution de l'ampleur (montant total) des ajustements que ces mesures représentent par rapport aux données conformes aux PCGR (Marques, 2006; Entwistle et al., 2006; Helfin et Hsu, 2008; Kolev et al., 2008; Pawlewicz, 2011). Quoique dans certaines de ces études les diminutions observées soient relativement mineures, elles doivent être mises en perspectives de la tendance historique inverse. En effet, dans les années qui ont précédé la mise en place de la réglementation, l'utilisation des MFHN avait connu une croissance impressionnante (Bradshaw et Sloan, 2002; Bhattacharya et al., 2004). Plus précisément, l'étude de Bradshaw et Sloan (2002) démontre que parmi un échantillon 
substantiel de communiqués publiés par des entités publiques au cours des années 1986 et 1987, $83 \%$ de ceux-ci ne présentaient que des mesures conformes aux PCGR alors que dans les années 1998 et 1999 , ce taux avait baissé à $28 \%$. Malgré ce qui précède, des études plus récentes et qui couvrent une période plus étendue suggèrent que l'impact de la réglementation n'aura été que temporaire du moins en ce qui concerne la fréquence de l'utilisation des MFHN. En réalité, et malgré la diminution observée durant les quelques années qui ont immédiatement suivi la mise en place de la réglementation, l'utilisation des MFHN aura connu une croissance soutenue depuis (Black et al., 2017; Brown et al., 2012b). Comme mentionné en introduction, la très grande majorité des entités composant les principaux indices nord-américains présentent maintenant, à un degré ou à un autre, des MFHN, en augmentation évidente par rapport à la situation qui prévalait seulement quelques années auparavant ${ }^{22}$.

Mais la fréquence d'utilisation des MFHN ne peut à elle seule démontrer l'efficacité de la réglementation, laquelle dans l'état actuel des choses n'a certes pas enrayé le phénomène des MFHN, mais pourra quand même en avoir amélioré la qualité. En fait, certains considèrent la diminution de la fréquence d'utilisation des MFHN comme étant un effet négatif de la réglementation (Bernstein, 2003). Comme les MFHN sont généralement considérées comme pertinentes du fait qu'elles ont une valeur ajoutée par rapport aux données financières traditionnelles, la mise en place d'une réglementation qui décourage les entités à publier ces informations, hormis si elles sont présentées dans une perspective opportuniste, ne sera pas considérée comme un succès. Ainsi, une réglementation efficace permettra de favoriser la publication d'information pertinente en limitant les comportements opportunistes des préparateurs et en donnant un sentiment de confiance aux utilisateurs.

Les principales études réalisées aux États-Unis après la mise en place de la réglementation $G$ démontrent qu'elle aura vraisemblablement eu des effets positifs sur la qualité des MFHN. Alors que le nombre de MFHN a diminué dans l'ensemble, les entités qui historiquement démontraient une faible relation (un écart important) entre le bénéfice mesuré selon les PCGR et le rendement (évalué par l'évolution du ratio cours/valeur comptable) et qui étaient alors identifiées comme poursuivant davantage un objectif de communication ont augmenté leur présentation de MFHN, laissant une diminution plus importante pour les entités qui adoptaient, avant la réglementation, des comportements plus opportunistes $(\mathrm{Yi}, 2012)$. Une conclusion similaire découle de l'observation d'une importante diminution dans le nombre et dans l'ampleur des ajustements présentés pour améliorer les résultats par rapport aux mesures PCGR ce que les auteurs interprètent comme une diminution du biais des gestionnaires dans la présentation des MFHN (Entwistle et al., 2006). Enfin, en considérant plus opportunistes les exclusions pour " autres éléments » par opposition aux exclusions pour " éléments exceptionnels », la réglementation aura également donné des résultats positifs en démontrant une diminution plus importante dans les ajustements de la première catégorie (Helfin et al., 2008). Les résultats de cette dernière étude peuvent toutefois être nuancés à la lumière d'une analyse portant spécifiquement sur la qualité des exclusions effectuées dans l'une ou l'autre de ces catégories (Kolev et al., 2008). En considérant qu'une relation négative entre les « exclusions » et les performances futures démontre une mauvaise qualité d'ajustements, en ce que ceux-ci représentent vraisemblablement des items récurrents, l'augmentation dans la qualité des ajustements pour «autres éléments » doit être considérée parallèlement à la diminution observée dans les ajustements pour " éléments exceptionnels », ce qui les amène à conclure dans les termes suivants :

While excluding "other" expenses from non-
GAAP earnings in the period prior to SEC inter-
vention was both relatively inexpensive and easily
concealed through a lack of disclosure, our results
suggest that managers have adapted to an alternate
reporting strategy in the post-SEC intervention
period by shifting more recurring items into special
items (Kolev et al., 2008, 177).

Plus spécifiquement, lorsque les organismes de réglementation comptables ont exigé la constatation en charge des rémunérations à base d'actions, plusieurs entités ont " ajusté » leur résultat pour exclure cette charge sous le prétexte qu'il ne s'agissait pas d'une dépense récurrente. À cet effet, Black et al. (2017, p. 235), qui observe également certains effets positifs de la réglementation, admettent que : 
"we conclude that even though regulation has influenced aggressive non-GAAP disclosures, a number of firms still appear to endorse aggressive non-GAAP exclusions. ». La réglementation, dans sa forme actuelle, n'aura donc pas sonné la fin de l'opportunisme. Les limites de la réglementation se font également sentir lorsque les études sont réalisées dans une perspective à plus long terme. Alors que les médias continuent de dénoncer les abus dans la publication des $\mathrm{MFHN}^{23}$, les études plus récentes appuient ces déclarations en démontrant qu'une décennie plus tard, les effets de la réglementation ont essentiellement disparu et qu'en fait la situation actuelle se serait même détériorée par rapport à celle qui prévalait avant la réglementation (Espahbodi et al., 2015).

En ce qui concerne la confiance des investisseurs dans les MFHN, il semble que la réglementation ait eu des résultats qui demeurent pour l'instant mitigés. La plupart des études présentées à ce niveau mesurent essentiellement la réponse des investisseurs aux annonces de résultats par les entités lesquelles sont constituées principalement de MFHN. Ainsi, si les investisseurs estiment l'annonce fiable, leur confiance dans l'information sera démontrée par un haut niveau de transaction durant la période suivant l'annonce. Alors que certains observent une augmentation de la confiance des utilisateurs envers les MFHN (Pawlewicz, 2011; Marques 2006) d'autres n'ont pu détecter aucun réel changement $(\mathrm{Yi}, 2012)$. Par ailleurs, une autre étude démontre qu'à la suite de la mise en place de la réglementation, les MFHN étaient moins utilisées aux fins des prévisions faites par les investisseurs démontrant vraisemblablement une diminution de la confiance envers de telles mesures (Heflin et Hsu, 2008).

\section{3 État actuel des lieux}

De toute évidence, la réglementation dans son état actuel n'aura pas découragé les entités à publier des MFHN. En fait pour certains, cette réglementation aura plutôt contribué, dans une certaine mesure, à la prolifération des MFHN à plus long terme :

Once companies received the blessing of regulators to use their own accounting metrics, however, the predictable happened: More and more companies used them, and used them to exclude more and more expenses ${ }^{24}$.

Les autorités nord-américaines ${ }^{25}$ auront donc opté pour une approche réglementaire relativement souple en ne cherchant ni à interdire ni à standardiser les MFHN et les succès d'une telle approche sont aujourd'hui clairement mitigés. Cette latitude aura effectivement un prix. En l'absence de mesures standards et définies, aucune comparaison n'est possible entre les entités ni d'ailleurs dans le temps, car les entités ont toujours l'entière liberté de redéfinir, d'un exercice à l'autre, la nature de leurs MFHN. Également, la discrétion absolue laissée aux directions quant aux ajustements effectués laisse encore beaucoup de place aux comportements opportunistes. Finalement, dans l'abondance déjà spectaculaire des informations financières fournies par les entités, toutes ces explications, conciliations et justifications pourront, tout au moins pour un lecteur non averti, noyer les données essentielles ou à la limite les rendre difficiles à trouver et à comprendre.

La situation actuelle est encore plus alarmante lorsqu'on considère que même les quelques exigences de présentation ne sont pas toujours respectées, et ce, sans réelles conséquences du moins au Canada où les autorités semblent effectivement plus conciliantes. Un article récent du Globe and Mail faisait effectivement état d'un rapport effectué par Veritas Investment Research Corp. dans lequel on dénonçait le fait qu'environ $35 \%$ des membres du S\&P/TSX 60 ne suivaient pas les recommandations de l'Autorité de marchés financiers en regard des $\mathrm{MFHN}^{26}$. Dans un récent rapport, l'ACVM constate que les informations présentées par les entités comportent, dans plus de $70 \%$ des cas, des lacunes lesquelles sont suffisamment importantes dans $20 \%$ des cas pour exiger un retraitement et la présentation de nouveaux documents ${ }^{27}$.

\section{Conclusion}

L'analyse de la situation actuelle nous aura permis de constater que la très grande majorité des MFHN sont liées à des mesures diverses de résultats. La littérature est par ailleurs quasi unanime quant à la pertinence de ces mesures alternatives, mais elle l'est tout autant quant à la présence toujours trop importante de comportements opportunistes, et ce, malgré les consignes données par les autorités des marchés financiers.

La réglementation des $\mathrm{MFHN}$, à l'instar de toutes lois, règles ou normes mises en place, est mise devant un choix fondamental quant au niveau de rigidité à imposer. D’un côté, une approche plus 
souple qui laisse aux directions d'entités une grande latitude favorisera l'identification de mesures adaptées au contexte spécifique. D'un autre côté, une approche plus directive et standardisée permettrait de limiter les comportements opportunistes en plus de fournir des données plus comparables et plus faciles à saisir pour les utilisateurs. La comparabilité est particulièrement importante dans le cadre des décisions d'investissement qui impliquent généralement un choix entre plusieurs entités.

Il faut par ailleurs envisager qu'à tort ou à raison, la profession comptable dont la mission première est la protection du public, sera tôt ou tard éclaboussée par les dérives des MFHN. En effet, l'investisseur moyen ne peut faire la différence entre les données tirées des états financiers audités et toutes les autres informations rendues publiques par des communiqués émanant des directions d'entités, mais aussi souvent reprises par les médias sans identification systématique des documents sources concernés et qui sont donc souvent considérées comme des vérités ou, du moins, perçues comme des données auditées au même titre que les états financiers traditionnels.

Dans ces circonstances, il nous apparait que la normalisation comptable pourrait être plus proactive dans ce débat. Évidemment, la profession pourrait laisser à d'autres le problème puisqu'il existe effectivement d'autres pistes de solution qui ont été identifiées pour améliorer la situation. Certaines études (Rashty et al., 2014, Seetharaman et al., 2014) semblent en effet démontrer une association positive entre la qualité des MFHN présentées et l'indépendance accrue du conseil d'administration, une plus grande expertise comptable sur les comités d'audit et un meilleur environnement de contrôle. Devant l'opportunisme actuellement constaté, nous ne pouvons qu être sceptiques quant à l'efficacité à long terme de ces solutions alternatives volontaires et subjectives. Quoi qu'il en soit, il nous semble que l'amélioration des états financiers traditionnels pourrait s'avérer une solution nettement plus simple, plus efficace et plus robuste à long terme. Plus spécifiquement, comme les principales MFHN sont en fait des données dérivées des détails actuellement cachés dans l'état des résultats, la présentation de cet état financier traditionnel pourrait être revue afin de mieux répondre aux besoins des investisseurs ${ }^{28}$. Sans pour autant mettre de côté le principe actuel du «bénéfice global unique $^{29}$ », la présentation des résultats pourrait être réorganisée de telle sorte à faire des calculs intérimaires de bénéfices pour faire ressortir les différents éléments recherchés par les utilisateurs.

Afin d'assurer une information bien ciblée, mais aussi facile à comprendre et à repérer, les composantes des résultats devraient être présentées obligatoirement dans un seul et unique état des résultats lequel pourrait alors présenter les éléments dans un ordre reflétant dans une certaine mesure la probabilité de récurrence, mais aussi le niveau de liquidité30. Sans révolutionner le monde de la comptabilité, il serait déjà possible de s'inspirer a priori de la structure actuelle du tableau des flux de trésorerie qui distingue assez facilement les activités opérationnelles, les activités d'investissement et les activités de financement. Une telle présentation des résultats permettra au lecteur de cibler les résultats attribuables aux activités opérationnelles normales en identifiant clairement les éléments non monétaires, les charges spécifiques liées à la structure de financement, les résultats qui ne sont pas liés aux activités courantes et qui pourraient inclure les items spéciaux, les éléments exceptionnels et même les charges constatées immédiatement, mais assumées dans une perspective à plus long terme (déboursés qui sans correspondre à la notion d'actif représente un potentiel d'avantages futurs). À l'analyse des « ajustements» fréquemment apportés dans les MFHN, l'approche «bilan » priorisée depuis quelques années par la normalisation comptable semble être en partie responsable du phénomène des MFHN, mais si la profession comptable ne veut pas faire marche arrière dans son approche fondamentale qui se justifie par ailleurs à plusieurs égards, elle pourra difficilement assurer sa propre pertinence à long terme. En effet, si les entités ne sont pas en mesure de présenter clairement les dépenses qu'elles ont encourues dans une perspective à long terme et qui, aux fins strictement comptables, ne peuvent être considérés comme des actifs dû aux risques qui y sont associés (par exemple, les frais de recherche, les frais de restructuration, la reconnaissance des services passés dans le cas des régimes de retraite), les règles comptables ne permettront pas d'atteindre l'objectif fondamental d'aider les utilisateurs à prendre des décisions éclairées.

Il nous semble évident qu'une présentation standard favorisera la comparabilité d'une entité à l'autre, mais 
aussi d'une période à l'autre. Comme toutes les informations seraient présentées dans un seul et même tableau, en l'occurrence l'état des résultats, les mesures tant convoitées seraient non seulement faciles à repérer, mais elles seraient tout aussi faciles à comprendre. Dans la perspective où la responsabilité première des états financiers incombe toujours à la direction des entités, son influence sur la classification et toute la flexibilité qui en découle ne devrait pas être compromise au premier abord. Toutefois, une telle présentation des résultats décomposés dans le corps même des états financiers et correspondant aux principales MFHN actuellement publiées présente en outre l'avantage d'être soumise au processus d'audit ${ }^{31}$ sans pour autant enlever toute la flexibilité laissée à la direction des entités dans la présentation de ses états financiers.

\section{NOTES}

1 Le paragraphe OB6 du Cadre conceptuel de l'information financière, Manuel de CPA Canada - Partie I est explicite à ce sujet « [...] les rapports financiers à usage général ne contiennent pas ni ne peuvent contenir toute l'information dont les investisseurs, les prêteurs et les autres créanciers actuels et potentiels ont besoin. Il faut donc que ces utilisateurs tiennent compte d'informations pertinentes provenant d'autres sources, par exemple des informations sur l'état général actuel et prévisible de l'économie, sur les événements et le climat politique, ou sur les perspectives d'avenir du secteur d'activité et de l'entreprise».

2 Un article récent du Wall Street Journal rapportait les données suivantes : “The adjusted, or customized, figures many finance chiefs use to supplement their company's standard financial reports inflate income by an average of $44 \%$ at profitable companies, according to new research by financial-data provider Calcbench Inc". Shumsky, T. et Francis, T. (2016, 28 juin). Accounting choices blur profit Picture, Wall Street Journal.

3 Tous les montants proviennent du rapport annuel 2016 de Bombardier, notamment aux pages 17 - Éléments spéciaux, 43 - Rapprochement du résultat net ajusté et du résultat net et 44 - Rapprochement du RAIIA ajusté et du RAII ajusté par rapport au RAII.

4 Selon une étude réalisée par Jack Ciesielski, CFA en 2015 et rapportée dans plusieurs quotidiens, 90 \% des compagnies composant le S\&P 500 utilisent maintenant une forme quelconque de MFHN (Shumsky et Francis, op. cit., note 2). Au Canada, le Globe \& Mail rapportait récemment que 59 des compagnies formant le S\&P/TSX60 faisaient de même. Qui plus est, ce même article avançait qu'au moins $80 \%$ des MFHN avaient un impact positif sur les mesures financières dont les principales étaient liées à la rentabilité de l'entité. Milstead, D. (2016, 24 sept.). How companies play with the books. Globe \& Mail, p. B9.

5 Les plus récentes révisions de normes comptables mettent effectivement en évidence cette « approche bilan » maintenant préconisée par les organismes de normalisation. À titre d'exemple, la norme sur les immobilisations incorporelles définit maintenant de façon très restrictive un tel actif en fonction d'une ressource économique qui aura été créée. Toute somme investie par une entité qui ne correspond pas à cette définition sera par défaut imputée aux résultats, et ce, nonobstant les retombées économiques à long terme qu'elle pourrait possiblement entraîner. Dans cette même perspective, la dernière mouture de la norme sur les impôts sur le bénéfice de même que celle sur la rémunération du personnel se concentrent maintenant uniquement sur la mesure de l'actif ou du passif inhérent, laissant par défaut aux résultats tout ce qui ne peut être intégré au bilan.

6 Dans une perspective comptable, la richesse d'une entité peut être définie par la mesure de l'ensemble des actifs nets dont elle dispose, soit le total de ses actifs (ressources économiques disponibles) moins le total de ses passifs (dettes). Dans le système comptable en partie double adopté depuis toujours à l'échelle universelle, le montant de ces actifs nets correspondra par ailleurs au montant de ses capitaux propres, soit le montant des capitaux investis par les actionnaires auquel s'ajoute les bénéfices accumulés et non distribués.

7 Paragraphe OB2 du Cadre conceptuel de l'information financière, Manuel de CPA Canada - Partie I «L'objectif de l'information financière à usage général est de fournir, au sujet de l'entité qui la présente (l'entité comptable), des informations utiles aux investisseurs, aux prêteurs et aux autres créanciers actuels et potentiels aux fins de leur prise de décisions sur la fourniture de ressources à l'entité. [...]».

8 Conformément au paragraphe 10 de la norme internationale d'information financière IAS1, Manuel de CPA Canada - Partie I, les états financiers à vocation générale comprennent généralement un état de la situation financière (bilan), un état du résultat net et des autres éléments du résultat global, un état des variations des capitaux propres de la période et un tableau des flux de trésorerie, cet ensemble étant généralement désigné comme le « corps des états financiers ». Toutefois, un jeu complet d'états financiers inclut également des notes qui fournissent un résumé des principales méthodes comptables et d'autres informations explicatives concernant les éléments présentés dans le corps des états financiers. 
9 Les utilités multiples des notes aux états financiers ont été officialisées dans un document publié par l'organisme de normalisation comptable canadien soit, Hugues J. et Fisher A. 2014, Lire les états financiers : Que me faut-il savoir?, CPA Canada, 2014.

10 À titre d'exemple, les notes complémentaires incluses dans les états financiers de Bombardier Inc. pour l'exercice financier terminé le 31 décembre 2016 totalisent 81 pages alors que le corps des états financiers tient sur 5 pages.

11 Conclusions tirées de documents de travail publiés par les autorités régissant les marchés financiers en Australie (FINSIA et AICD), soit Underlying profit: A discussion paper on the reporting of non-statutory profit information - Discussion paper (2008) et Underlying profit: Principles for the reporting of nonstatutory profit information, Policy Guidance paper (2009).

12 Extrait de l'avis 52-306 de l'ACVM publié le 14 janvier 2016, page 2.

13 Site internet de l'ESMA. Repéré à https://www.iasplus.com/en/news/2014/02/esma-apm

14 Hoogervorst, H. (2014, 5 février), Defining Profit or Loss and OCI... can it be done? Allocution présentée à Tokyo.

15 Outre la pertinence (qui sous-entend une valeur de prévision et une valeur de confirmation) et la fidélité, le chapitre 3 du Cadre conceptuel de l'information financière, Manuel de CPA Canada - Partie I, considère qu'une information financière est de qualité lorsqu'elle est compréhensible, comparable, vérifiable et présentée en temps opportun.

16 La situation n'est certes pas facilitée par la réglementation actuelle qui exige, en accompagnement des MFHN, plusieurs explications, justifications et réconciliations (voir la section spécifique sur la réglementation).

17 Les ajustements « agressifs » sont ceux qui sont généralement considérés comme récurrents par leur nature et leur fréquence.

18 SEC, Accounting Series Release No. 142 - Reporting Cash Flow and Other Related Data, 1973.

19 Cette loi aussi appelée « Public Company Accounting Reform and Investor Protection Act of 2002 » vise, comme son nom l'indique, à améliorer la divulgation des informations financières des sociétés cotées et à protéger par le fait même les investisseurs.

20 Vise spécifiquement le Regulation G (applicable à toute communication publique), mais aussi les Item 10 of Regulation S-K et Item 10 of Regulation S-B (applicable pour tous les documents devant être produits auprès de la SEC.)

21 À titre d'exemple, les conciliations et explications des MFHN dans le rapport annuel 2016 de Bombardier représentent près du tiers du rapport de gestion.

22 Selon une étude réalisée par Jack Ciesielski, CFA en 2015 et rapportée dans plusieurs quotidiens, 448 des entités composant l'indice S\&P500 présentaient des MFHN en 2015 alors que ce nombre n'était que de 358 en 2009, ce qui représente une augmentation de $25 \%$ (Shumsky et Francis, op. cit., note 2). La situation est similaire au Canada alors qu'en 2004 seulement une poignée d'entités du TSX60 affichait des MFHN alors qu'en 2015, seulement une de ces entités ne présentait pas de telles mesures (Milstead, op. cit., note 4).

23 Shumsky et Francis, op. cit., note 2 et Milstead, op. cit., note 4.

24 Milstead, op. cit., note 4.

25 En fait, aucune juridiction dans le monde n'a choisi de réglementation plus stricte à l'exception de l'Afrique du Sud qui impose la présentation d'une mesure alternative de bénéfice dans le corps de ses états financiers (Venter et al. 2014).

26 Milstead, op. cit., note 4.

27 FAIR Canada (2006, juin). Mesures non conformes aux PCGR : des outils utiles pour les investisseurs ou un non-sens néfaste?

28 La norme internationale d'information financière IAS1, Manuel de CPA Canada - Partie I est actuellement très peu directive en matière d'exigences de présentation, laissant place à beaucoup de confusion en regard de l'identification même de ce que constitue une MFHN. Cette préoccupation a d'ailleurs été clairement exprimée récemment dans les termes suivants : «Unlike many of the accounting standards that it replaced, IFRS does not impose binding formats for the presentation of financial information. Instead, it requires that, at a minimum, certain line items must be presented on the face of the financial statements, but allows additional line items to be presented where relevant to an understanding of the issuer's financial position and performance. Because not all of the line items that an issuer may include in its financial statements are specified by IFRS, it is more difficult to say that a particular measure is a non-Gaap item, given that one issuer may include it in its financials and another may not." (Capeci, 2010, p. 3).

29 Incluant les « autres éléments du résultat global » qui, à notre avis, ne devraient pas faire l'objet d'un état distinct étant donné le niveau de complexité qu'il implique et en conséquence toute la confusion qu'il entraîne, laissant peu de place à une valeur ajoutée. 
30 Comme mentionné précédemment, une présentation par voie de notes complémentaires ne serait pas une solution optimale puisque la littérature démontre clairement que ces dernières n'ont pas une valeur informationnelle équivalente au corps même des états financiers.

31 Certains pourraient juger qu'un tel audit, qui serait en grande partie liée au jugement par un professionnel comptable des perspectives des dirigeants d'entités pourrait donner un faux sens de sécurité aux utilisateurs. À cet effet, le commentaire suivant est à considérer : "Veritas has some recommendations for how Canadian regulators should deal with the wave of new accounting measures being crafted, and some practices companies could engage in to make them better. One may be counterintuitive: Veritas believes a company's auditors should not be responsible for reviewing any measures that don't comply with generally accepted accounting principles. When an auditor signs off on "adjusted net income" that pumps up a company's profitability, it "has the potential to create a false sense of security," Veritas writes. If the auditor reviews the metric, Veritas says, investors may view the number as correct". Milstead, op. cit., note 4.

\section{RÉFÉRENCES}

Aubert, F. et Grudnitski, G. (2014). The role of reconciliation quality in limiting mispricing of non-GAAP earnings announcements by EURO STOXX firms. Advances in Accounting, 30(1), 154-167.

Barker, R. et Imam, S. (2008). Analysts' perceptions of 'earnings quality'. Accounting and Business Research, 38(4), 313-329.

Barth, M. E., Elliott, J. A. et Finn, M. W. (1999). Market rewards associated with patterns of increasing earnings. Journal of Accounting Research, 37(2), 387-413.

Bartov, E., Givoly, D. et Hayn, C. (2002). The rewards to meeting or beating earnings expectations. Journal of Accounting \& Economics, 33(2), 173-204.

Berger A. et Udell G. (1995). Relationship lending and lines of credit in small firm finance. Journal of Business, 68(3), 351-382.

Bernstein, D. (2003). SEC Adopts Rules for Non-GAAP Information. International Financial Law Review, 22(3), 39.

Bhattacharya, N., Black, E. L., Christensen, T. E. et Larson, C. R. (2003). Assessing the relative informativeness and permanence of pro forma earnings and GAAP operating earnings. Journal of Accounting and Economics, 36(1-3), 285-319.

Bhattacharya, N., Black, E. L., Christensen, T. E., et Mergenthaler, R. D. (2004). Empirical evidence on recent trends in pro forma reporting. Accounting Horizons, 18(1), 27-43.

Bhattacharya, N., Black, E., Christensen, T. et Mergenthaler, R. (2007). Who trades on pro forma earnings information? The Accounting Review, 82(3), 581-619.

Black, D. et Christensen, T, (2009). US managers use of 'pro forma' adjustments to meet strategic earnings targets. Journal of Business Finance and Accounting, 36(3-4), 297-326.

Black, E. L., Christensen, T. E., Kiosse, P. V. et Steffen, T. D. (2017). Has the regulation of non-GAAP disclosures influenced managers' use of aggressive earnings exclusions? Journal of Accounting, Auditing \& Finance, 32(2), 209-240.

Bradshaw, M. et Sloan, R. (2002). GAAP versus the street: an empirical assessment of two alternative definitions of earnings. Journal of Accounting Research. 40(1), 41-66.

Brooks R., Scott J. et Pearson T. (1996). The use of fair-value disclosures to assess liquidity et solvency in credit decision. Commercial lending review, 67-73.

Brown, L. et Sivakumar, K. (2003). Comparing the value relevance of two operating income measures. Review of Accounting Studies, 8(4), 561-572.

Brown, N. C., Christensen, T. E. et Elliott, W. B. (2012). The timing of quarterly 'pro forma' earnings announcements. Journal of Business Finance \&o Accounting, 39(3-4), 315-359.

Brown, N. C., Christensen, T. E., Elliott, W. B. et Mergenthaler, R. D. (2012). Investor sentiment and pro forma earnings disclosures. Journal of Accounting Research, 50(1), 1-40.

Burgstahler, D. et Dichev, I. (1997). Earnings management to avoid earnings decreases and losses. Journal of Accounting \& Economics, 24(1), 99-126.

Capeci, B. (2010). Listen to Cesr. International Financial Law Review, 29(5), 54-56. 
Demirakos, E. G., Strong, N. C. et Walker, M. (2004). What valuation models do analysts use? Accounting Horizons, 18(4), 221-240.

Doyle, J. T., Jennings, J. N. et Soliman, M. T. (2013). Do managers define non GAAP earnings to meet or beat analyst forecasts? Journal of Accounting \& Economics, 56(1), 40-56.

Elliott, W. B. (2006). Are investors influenced by pro forma emphasis and reconciliations in earnings announcements? The Accounting Review, 81(1), 113-133.

Entwistle, G. M., Feltham, G. D. et Mbagwu, C. (2006). Financial reporting regulation and the reporting of pro forma earnings. Accounting Horizons, 20(1), 39-55.

Espahbodi, H., Espahbodi, P. et Espahbodi, R. (2015). Did analyst forecast accuracy and dispersion improve after 2002 following the increase in regulation? Financial Analysts Journal, 71(5), 20-37.

Fernandez, A. (2000). Les nouveaux tableaux de bord des décideurs (2e édition). Paris, France : Éditions d'Organisation.

Frederickson, J.R. et Miller, J.S. (2004). The effects of pro forma earnings disclosures on analysts' and nonprofessional investors' equity valuation judgments. The Accounting Review, 79(3), 667-686.

Graham, J. R., Harvey, C. R. et Rajgopal, S. (2006). Value destruction and financial reporting decisions. Financial Analysts Journal, 62(6), 27-39.

Graham, J. R., Harvey, C. R. et Rajgopal, S. (2004). The economic implications of corporate financial reporting. Cambridge, MA: National Bureau of Economic Research.

Harper R., Mister W. et Strawser J. (1987). The impact of new pension disclosure rules on perceptions of debt. Journal of Accounting Research, 25(2), 327-330.

Heflin, F. et Hsu, C. (2008). The impact of the SEC's regulation of non-GAAP disclosures. Journal of Accounting \& Economics, $46(2), 349$.

Hirst, E. et Hopkins, P. (1998). Comprehensive income reporting and analysts' valuation judgments. Journal of Accounting Research, 36, 47-75.

Hirst, E., Hopkins, P. et Wahlen, J. (2004). Fair values, income measurement, and bank analysts' risk and valuation judgments. The Accounting Review, 79(2), 453-472.

Holtzman, M., Fonfeder R. et Yun, J.K. (2003).Goodbye "pro forma" earnings". Strategic Finance, 85(5), 33-35.

Kemp, R., Overstreet, Jr. et George, A. (1990). A study of the information needs of commercial loan officers. The Journal of Commercial Bank Lending, 72, 47.

Kolev, K., Marquardt, C. A. et McVay, S. E. (2008). SEC scrutiny and the evolution of non-GAAP reporting. The Accounting Review, 83(1), 157-184.

Kwoz H. (2002). The effect of cash flow statement format on lenders' decisions. The International Journal of Accounting, 37(3), $347-362$.

Lougee, B. A. et Marquardt, C. A. (2004). Earnings informativeness and strategic disclosure: an empirical examination of "pro forma" earnings. The Accounting Review, 79(3), 769-795.

Malone, L., Tarca, A. et Wee, M. (2016). IFRS non-GAAP earnings disclosures and fair value measurement. Accounting \& Finance, 56(1), 59-97, doi : 10.1111/acfi.12204.

Marques, A. (2006). SEC interventions and the frequency and usefulness of non-GAAP financial measures. Review of Accounting Studies, 11(4), 549-574.

Pawlewicz, R. J. (2011). The economic consequences of disclosure regulation: evidence from regulation G (Order No. 3478773). Available from ProQuest Dissertations \& Theses Global : Business. (903968427).

Rashty, J. et O’Shaughnessy, J. (2014). Non-GAAP earnings and board independence. CPA Journal, 84(3), 36-39.

Scott, J.A. (2006). Loan officer turnover and credit availability for small firms. Journal of Small Business Management, 44(4), 544-562.

Seetharaman, A., Wang, X. et Zhang, S. (2014). An empirical analysis of the effects of accounting expertise in audit committees on non-GAAP earnings exclusions. Accounting Horizons, 28(1), 17-37. 
Skinner, D. J. et Sloan, R. G. (2002). Earnings surprises, growth expectations, and stock returns or don't let an earnings torpedo sink your portfolio. Review of Accounting Studies, 7(2-3), 289-312.

Venter, E. R., Emanuel, D. et Cahan, S. F. (2014). The value relevance of mandatory non-GAAP earnings. Abacus, 50(1), 1-24.

Viger, C., Belzile, R. et Lagrange B. (2005). L'effet du mode de divulgation des options d'achat d'actions sur les décisions des directeurs de comptes de Desjardins. Les séminaires de la chaire de coopération Guy-Bernier, février 2006.

Yi, H. (2012). Has regulation G improved the information quality of non-GAAP earnings disclosures? Seoul Journal of Business, $18(2), 95-145$.

Zhang, H. et Zheng, L. (2011). The valuation impact of reconciling pro forma earnings to GAAP earnings. Journal of Accounting and Economics, 51(1-2), 186-202. 